\title{
Self-consistent electronic structure and segregation profiles of the Cu-Ni (001) random-alloy surface
}

Ruban, Andrei; Abrikosov, I. A.; Kats, D. Ya.; Gorelikov, D.; Jacobsen, Karsten Wedel; Skriver, Hans Lomholt

Published in:

Physical Review B

Link to article, DOI:

10.1103/PhysRevB.49.11383

Publication date:

1994

Document Version

Publisher's PDF, also known as Version of record

Link back to DTU Orbit

Citation (APA):

Ruban, A., Abrikosov, I. A., Kats, D. Y., Gorelikov, D., Jacobsen, K. W., \& Skriver, H. L. (1994). Self-consistent electronic structure and segregation profiles of the Cu-Ni (001) random-alloy surface. Physical Review B, 49(16), 11383-11396. https://doi.org/10.1103/PhysRevB.49.11383

\section{General rights}

Copyright and moral rights for the publications made accessible in the public portal are retained by the authors and/or other copyright owners and it is a condition of accessing publications that users recognise and abide by the legal requirements associated with these rights.

- Users may download and print one copy of any publication from the public portal for the purpose of private study or research.

- You may not further distribute the material or use it for any profit-making activity or commercial gain

- You may freely distribute the URL identifying the publication in the public portal 


\title{
Self-consistent electronic structure and segregation profiles of the $\mathrm{Cu}-\mathrm{Ni}(001)$ random-alloy surface
}

\author{
A. V. Ruban \\ Chair for Theoretical Physics, Moscow Institute for Steel and Alloys, Moscow, Russia \\ I. A. Abrikosov \\ Center for Atomic-Scale Materials Physics and Physics Department, Technical University of Denmark, \\ DK-2800 Lyngby, Denmark \\ and Condensed Matter Theory Group, Physics Department, Uppsala University, S-75121 Uppsala, Sweden \\ D. Ya. Kats and D. Gorelikov \\ Chair for Theoretical Physics, Moscow Institute for Steel and Alloys, Moscow, Russia \\ K. W. Jacobsen and H. L. Skriver \\ Center for Atomic-Scale Materials Physics and Physics Department, Technical University of Denmark, \\ DK-2800 Lyngby, Denmark
}

(Received 2 November 1993; revised manuscript received 14 January 1994)

\begin{abstract}
We have calculated the electronic structure and segregation profiles of the (001) surface of random $\mathrm{Cu}-\mathrm{Ni}$ alloys with varying bulk concentrations by means of the coherent potential approximation and the linear muffin-tin-orbitals method. Exchange and correlation were included within the localdensity approximation. Temperature effects were accounted for by means of the cluster-variation method and, for comparison, by mean-field theory. The necessary interaction parameters were calculated by the Connolly-Williams method generalized to the case of a surface of a random alloy. We find the segregation profiles to be oscillatory with a strong preference for $\mathrm{Cu}$ to segregate towards the surface of the alloy.
\end{abstract}

\section{INTRODUCTION}

Ever since Gibbs predicted the effect of surface segregation at the turn of the 19th century, evidence for the existence of this most important surface phenomenon has been accumulating as may be seen, for instance, in the survey by Johnson and Blakely. ${ }^{1}$ In particular, a substantial amount of work has been devoted to the investigation of surface segregation in random alloys of $\mathrm{Cu}$ and $\mathrm{Ni}$, owing to the effect on the catalytic properties of this alloy system. Based on these investigations, the consensus is that copper has a strong tendency to segregate towards the surface of the alloy at all bulk concentrations and over a wide range of temperatures.

Although one may safely conclude that $\mathrm{Cu}$ segregates to the surface of a $\mathrm{Cu}-\mathrm{Ni}$ alloy, the actual shape of the segregation profile is still under discussion. In particular, one would like to know whether the concentration oscillates as one moves away from the surface. Unfortunately, the experimental data as well as the theoretical evidence appear to lead to conflicting conclusions. One problem is that the experimental determination of a concentration profile is a very difficult task. ${ }^{2}$ Moreover, some techniques rely on indirect evidence in order to obtain the concentration at subsurface layers which may cast some doubt on the results. With respect to the theoretical investigations of segregation profiles such undertakings require time-consuming and accurate calculations of total ener- gies. As a result one typically introduces a number of simplifying assumptions whereby the conclusions drawn from the results may be somewhat unreliable.

In Table I we have summarized the available information concerning the shape of the segregation profile in $\mathrm{CuNi}$ alloys ${ }^{3-17}$ and from this compilation one may notice certain tendencies. Thus it appears that the experiments which predict a monotonic concentration profile are based on indirect evidence. Moreover, Watanabe et al. ${ }^{3}$ for example, assumed a monotonic concentration profile in order to interpret their experimental data. The problems which occur in an indirect analysis may also be illustrated by the example of Ling et al. ${ }^{5}$ These authors predicted an oscillatory segregation profile on the basis of the relative intensities of the $\mathrm{Cu}$ and $\mathrm{Ni}$ peaks in the measured ultraviolet photoelectron spectra. Later, however, Durham et al. ${ }^{18}$ showed that this effect could be caused by the surface electronic structure rather than by the composition of the subsurface layers. In contrast, it also appears from Table I that the two experiments which may be regarded as direct predict an oscillatory $\mathrm{Cu}$ concentration.

The results of the theoretical calculations shown in Table $I$ are also at variance among each other. In this case it may be significant to note that the methods which are based on the one-electron contribution to the total energy predict a monotonic segregation profile whereas the calculations which are based on the minimization of the complete total energy of the system lead to an oscilla- 
TABLE I. The shape of the segregation profile in $\mathrm{Cu}-\mathrm{Ni}$ systems obtained by different experimental and theoretical techniques.

\begin{tabular}{|c|c|c|c|c|c|}
\hline Method $^{\mathrm{a}}$ & $\begin{array}{l}\text { Concentration } \\
\text { depth profile }\end{array}$ & $\begin{array}{l}c_{\text {bulk }}^{\mathrm{Cu}} \\
\text { at. \% }\end{array}$ & Sample & Comments & Ref. \\
\hline $\mathrm{AES}^{\mathrm{b}}$ & monotonic & $0-100$ & polycrystal & indirect analysis & 3 \\
\hline $\mathrm{AES}^{\mathrm{b}}$ & monotonic & 0-100 & polycrystal & indirect analysis & 4 \\
\hline $\mathrm{UPS}+\mathrm{AES}^{\mathrm{b}}$ & oscillatory & 10 & $(110)$ & indirect analysis & 5 \\
\hline FIM $^{\mathrm{b}}$ & oscillatory & 5 & $(111)$ & direct experiment & 6,7 \\
\hline $\mathrm{XPS}+\mathrm{AES}^{\mathrm{b}}$ & oscillatory? & 5,50 & $(100),(111),(110)$ & indirect analysis & 8 \\
\hline ISS $^{\mathrm{b}}$ & oscillatory & 40 & polycrystal & direct experiment & 9 \\
\hline $\mathrm{RSM}^{\mathrm{c}}$ & oscillatory & 5 & $(111)$ & with relaxation & 7 \\
\hline $\mathrm{QC}^{\mathrm{c}}$ & monotonic & $0-100$ & $(100),(111)$ & without relaxation & 10 \\
\hline $\mathrm{QC}^{\mathrm{c}}$ & oscillatory & $0-100$ & $(100),(111)$ & with relaxation & 10 \\
\hline $\mathrm{MC}+\mathrm{EAM}^{\mathrm{c}}$ & oscillatory & $0-100$ & $(100),(111),(110)$ & total energy & 11 \\
\hline TBIM $^{\mathrm{c}}$ & monotonic & $0-100$ & (111) & one-electron energy & 12 \\
\hline $\mathrm{MC}^{\mathrm{c}}$ & monotonic & $0-100$ & $(100),(111),(110)$ & one-electron energy & 13 \\
\hline $\mathrm{MC}+\mathrm{EAM}^{\mathrm{c}}$ & oscillatory & 60 & $(100)$ & total energy & 14 \\
\hline $\mathrm{FESM}+\mathrm{EAM}^{\mathrm{c}}$ & oscillatory & $0-100$ & $(100)$ & total energy & 15 \\
\hline $\mathrm{GPM}+\mathrm{BW}^{\mathrm{c}}$ & monotonic & 25,75 & $(100)$ & one-electron energy & 16 \\
\hline $\mathrm{GPM}+\mathrm{MC}^{\mathrm{c}}$ & monotonic & $0-100$ & $(100)$ & one-electron energy & 17 \\
\hline
\end{tabular}

${ }^{a}$ Auger electron spectroscopy (AES); ultraviolet-photoelectron spectroscopy (UPS); time-of-flight atom probe field-ion microscopy (FIM); regular solution model (RSM); x-ray photoelectron spectroscopy (XPS); quasichemical approach (QC); ion-scattering spectroscopy (ISS); Monte Carlo simulations (MC); embedded atom method (EAM); tight-binding Ising model (TBIM); free-energy simulations method (FESM); general perturbation method (GPM); Bragg-Williams method (BW). ${ }^{\mathrm{b}}$ Experiments.

${ }^{\mathrm{c}}$ Calculations.

tory segregation profile for the (001) surface of $\mathrm{Cu}-\mathrm{Ni}$ alloys. It therefore appears that further calculations of the shape of the segregation profile in these alloys are highly desirable.

It is the aim of this work to perform local density functional calculations of the electronic structure and the segregation profile for the (001) surface of random $\mathrm{Cu}-\mathrm{Ni}$ alloys in the complete concentration range. For this purpose we employ the self-consistent linear-muffintin-orbitals coherent-potential (LMTO-CPA) technique within the atomic sphere approximation (ASA), which is based on the work of Andersen and co-workers. ${ }^{19-26}$ The present implementation of the method is described in Ref. 27 , where it was used to calculate properties of surfaces of uniform, unsegregated alloys. To calculate segregation profiles and phase equilibria in alloys at finite temperatures the LMTO-CPA technique must be supplemented by the methods of statistical mechanics which allow the determination of the free energy and the configurational entropy. The basic feature of these methods is their phenomenological treatment of the interatomic interactions in terms of interaction parameters or effective potentials of Ising-like Hamiltonians which may be obtained from first-principles total energy calculations.

At present there exist two different schemes which may be used to obtain the interatomic interaction parameters: the Connolly-Williams method ${ }^{28}$ (CWM) and the direct configurational averaging ${ }^{29}$ represented, for instance, by the widely used generalized perturbation method (GPM). ${ }^{30}$ The main advantage of the GPM is its ability to express the effective interaction parameters in an explicit and physically transparent form. This prop- erty of the GPM is especially convenient in calculations of interaction parameters in inhomogeneous semi-infinite systems, such as surfaces of alloys, because even in this case the expression for the effective potentials change their form only slightly. The GPM has recently been applied to the segregation problem by Dreysse et al. ${ }^{16}$ in the framework of tight-binding CPA and by Pasturel et al. ${ }^{17}$ within the more sophisticated LMTO-CPA technique.

While the GPM appears to be useful in the determination of effective interaction potentials they turn out to be inconvenient in actual calculations of phase equilibria in inhomogeneous systems. First of all, by definition the GPM only takes into account changes in the one-electron contribution to the total energy and does not account for the direct electrostatic interaction between atoms caused by charge-transfer effects. Therefore, the method is not directly applicable to a large group of systems. Second, since the effective potentials of the GPM are determined on the basis of results for systems described by definite external parameters such as volume and concentration, they have a corresponding explicit dependence on these parameters. In the case of an inhomogeneous system the number of such parameters increases appreciably. For instance, in the determination of the equilibrium concentration profile for a surface the GPM potentials depend explicitly on the concentration in each surface layer and hence one must map out the GPM potentials over large portions of this multidimensional space.

To avoid these problems we have in the present work determined the interatomic interactions on the basis of the Connolly-Williams procedure. ${ }^{28}$ In spite of the fact 
that the multisite interactions obtained in the CWM have no explicit form and are not even uniquely defined, the CWM has been widely applied in first-principles calculations of phase diagrams of real systems and it has been used successfully together with CPA total-energy calculations in a study of disordered $\mathrm{Ni}-\mathrm{Al}$ alloys. ${ }^{31}$ For the present purpose we generalize the CWM to treat the case of an fcc (001) surface which will allow us to calculate the concentration profile on the basis of the cluster variation method (CVM). Since the Connolly-Williams method takes into account the difference in the total energy of various compositions of the alloy surface it turns out to be more reliable in the calculation of interaction parameters than the methods which rely only on the oneelectron term.

\section{SURFACE ENERGY OF AN ALLOY WITH SEGREGATION}

The energy $\gamma$ required to form a surface may be calculated as the difference between the grand potential of a semi-infinite system of $N$ atoms containing the surface and the grand potential of the corresponding homogeneous system with the same number of atoms, i.e.,

$$
\gamma=\Omega-\Omega_{\text {bulk }}
$$

where the grand potential for a two-component system is given by

$$
\Omega=E-T S-\sum_{i=1,2} \mu_{i} N_{i}
$$

in terms of the internal energy $E$, the temperature $T$, the entropy $S$, the total number of the $i$ th species in the system $N_{i}$, and the chemical potential $\mu_{i}$ of the $i$ th species.

If we assume a particular segregation profile by specifying the concentration of the surface layers $c_{\Lambda}$, the surface energy at the temperature $T=0$ may be found from (2) to be

$E_{\text {surf }}=\sum_{\Lambda=m}^{M}\left[E_{\Lambda}^{\mathrm{ASA}}-E_{\Lambda}^{\mathrm{bulk}}\right]-\mu_{1} \sum_{\Lambda=1}^{M}\left[c_{\Lambda}-c_{\mathrm{bulk}}\right]$

where $\mu_{1}=-\mu_{2}$ for a two-component system. In (3) $E_{\Lambda}^{\mathrm{ASA}}$ is the partition of the total energy into layer contributions as allowed by the ASA, the sum in the first term is over the vacuum region $m \leq \Lambda \leq 0$ as well as over the alloy region $1 \leq \Lambda \leq M$, and $E_{\Lambda}^{\text {bulk }}$ is zero in the vacuum region and otherwise equal the total energy per atom of the bulk with concentration $c_{\text {bulk }}$.

In the LMTO-CPA total-energy calculations we have included two layers of empty spheres, i.e., $m=-1$, to simulate vacuum, and four layers of alloy, i.e., $M=4$, in the self-consistency procedure. This may partly be motivated by the fact that the state density of the third layer as discussed in Sec. VI is practically identical to the bulk state density. Since, in all the cases considered here, the contribution to the surface energy from the fourth layer,
$E_{4}^{\mathrm{ASA}}-E^{\text {bulk }}$, is less than $1 \mathrm{mRy}$ per atom, or $35 \mathrm{~mJ} / \mathrm{m}^{2}$, the cutoff at $M=4$ is expected to lead to absolute errors of this order. However, the error in the difference between surface energies which is the important quantity in the present application is typically much smaller than $1 \mathrm{~mJ} / \mathrm{m}^{2}$.

If we consider an infinitely large region the last term in (3) goes to zero. However, with $M=4$ the number of particles within the surface region is not conserved and hence the term must be included in the calculation of surface energies for alloys with segregation. In contrast to the case of a pure surface $e^{26,32}$ or the surface of a uniformly random alloy, ${ }^{27}$ one must know not only the total energy of the bulk but also the chemical potential. This we find from

$$
\mu_{1}=\left.\frac{d E_{\text {bulk }}}{d c}\right|_{c_{\text {bulk }}}
$$

i.e., as the concentration derivative of the bulk total energy. Finally, in the case of a disordered alloy $E_{\Lambda}$ and $E_{\text {bulk }}$ may be substituted by the single-site CPA expression $^{33}$

$$
E_{\text {alloy }}=c E_{1}+(1-c) E_{2}
$$

where the energy of the $\mathrm{Cu}$ and $\mathrm{Ni}$ atoms are denoted $E_{1}$ and $E_{2}$, respectively.

\section{CLUSTER-VARIATION METHOD FOR fcc (001) SURFACES}

In this section we present the cluster variation method in a form which may be used to treat the case of an fcc (001) surface of a random alloy. The CVM was first formulated for this case within the tetrahedron approximation by Kumar and Bennemann, ${ }^{34}$ who used it to calculate the order-disorder transition in the (001) surface of a $\mathrm{Cu}-\mathrm{Au}$ alloy. Here we combine their formulation with that of Kikuchi and Cahn, ${ }^{35}$ who developed the CVM to treat (001) antiphase and interphase boundaries in fcc based alloys. In addition, we shall take account of the renormalization of the interaction parameters occurring near the surface and introduce an additional term into the CVM Hamiltonian which describes the interaction between atoms in the surface layer and those of the next-nearest-neighbor layer.

Consider the semi-infinite structure which defines an (001) surface of an fcc lattice and let the layers parallel to the surface be numbered in increasing order from the surface layer and into the bulk. Let all sites in each layer be equivalent, i.e., neglect long-range order effects and let layer number $\Lambda=M+1$ be considered a bulk layer, so that the chemical potential and other thermodynamic properties of this and succeeding layers are considered to have reached the bulk values.

Within our model the basic cluster is the tetrahedron formed by two points in a particular layer and two points in the nearest-neighbor layer as illustrated in Fig. 1. Based on a single point in a given layer there are four 
a.
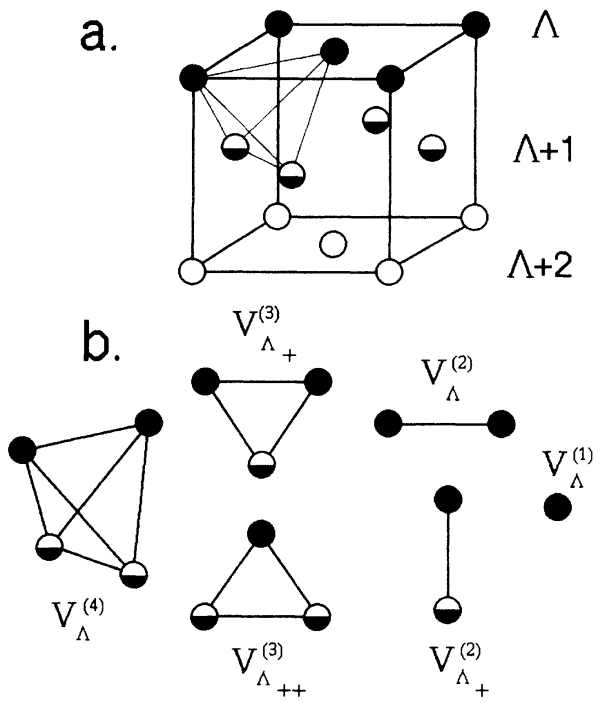

FIG. 1. (a) The tetrahedron of the four closest neighbors belonging, according to the present convention, to (001) plane $\Lambda$ in the fcc lattice. (b) The six different types of interactions, defined on this tetrahedron. The filled, half-filled, and open circles denote the atoms of the layers $\Lambda, \Lambda+1$, and $\Lambda+2$ planes, respectively.

geometrically equivalent tetrahedra above the layer and four equivalent tetrahedra below the layer with the exception of the surface layer which have only four tetrahedra pointing towards the bulk. All tetrahedra are labeled according to the number $\Lambda$ of the layer closest to the surface, $\mathrm{Cu}$ atoms are labeled 1 , and $\mathrm{Ni}$ atoms 2 . With these definitions we introduce the basic variable $z_{i j k l}^{\Lambda}$ of the method which designates the probability that the configuration $(i, j, k, l)$, where the first pair of indices refer to atoms in the layer $\Lambda$ and the second pair to atoms in the layer $\Lambda+1$, appears in the specified tetrahedron.

The system has two kinds of pair clusters, i.e., those connecting layers and those situated within layers. We therefore introduce the pair correlation $v_{i k}^{\Lambda}$ for pairs connecting the layers $\Lambda$ and $\Lambda+1$ and $y_{i j}^{\Lambda}$ for pairs within the $\Lambda$ th layer. The probability of finding atom $i$ in the $\Lambda$ th layer is defined as $x_{i}^{\Lambda}$ and this number corresponds to the composition within the layer. Because of geometrical constraints the variables are related by the expressions

$$
y_{i j}^{\Lambda}=\sum_{k, l} z_{i j k l}^{\Lambda}, \quad x_{i}^{\Lambda}=\sum_{j, k, l} z_{i j k l}^{\Lambda},
$$

and, in addition, they obey the normalization constraint

$$
\sum_{i, j, k, l} z_{i j k l}^{\Lambda}=1
$$

and the continuity relation

$$
\sum_{i, j} z_{i j k l}^{\Lambda}=\sum_{m, n} z_{k l m n}^{\Lambda+1} \quad, \quad 2 \leq \Lambda \leq M
$$

for all values of the indices $(k, l)$.

If the number of sites in a layer is $N$ the internal energy of the system may be written as the sum over layers of tetrahedron interactions

$$
E=2 N \sum_{\Lambda} \sum_{i, j, k, l} \varepsilon_{i j k l}^{\Lambda} z_{i j k l}^{\Lambda},
$$

where $\varepsilon_{i j k l}^{\Lambda}$ is a four-body interaction parameter that may be expressed in terms of one-, two-, three-, and four-site interactions calculated by the Connolly-Williams method to be described in Sec. IV. The factor of 2 reflects the fact that, although each site in a given layer defines four tetrahedra, each tetrahedron is shared by two sites and hence double counted in the sum over $\Lambda$.

The configurational entropy of the system may be given by ${ }^{34,36}$

$$
\begin{aligned}
S=k_{B} N\{ & -2 \sum_{i, j, k, l} L\left(z_{i j k l}^{(1)}\right)+\sum_{i, j} L\left(y_{i j}^{(2)}\right)+\left[\sum_{i, k} L\left(v_{i k}^{(1)}\right)+\sum_{i, l} L\left(v_{i l}^{(1)}\right)+\sum_{j, k} L\left(v_{j k}^{(1)}\right)+\sum_{j, l} L\left(v_{j l}^{(1)}\right)\right] \\
& \left.-\frac{1}{2}\left[\sum_{i} L\left(x_{i}^{(1)}\right)+\sum_{j} L\left(x_{j}^{(1)}\right)\right]-\frac{5}{4}\left[\sum_{k} L\left(x_{k}^{(2)}\right)+\sum_{l} L\left(x_{l}^{(2)}\right)\right]-\frac{1}{2}\right\} \\
+k_{B} N \sum_{\Lambda=2}\{ & -2 \sum_{i, j, k, l} L\left(z_{i j k l}^{\Lambda}\right)+\sum_{i, j} L\left(y_{i j}^{\Lambda}\right)+\sum_{k, l} L\left(y_{k l}^{\Lambda+1}\right)+\sum_{i, k} L\left(v_{i k}^{\Lambda}\right)+\sum_{i, l} L\left(v_{i l}^{\Lambda}\right)+\sum_{j, k} L\left(v_{j k}^{\Lambda}\right)+\sum_{j, l} L\left(v_{j l}^{\Lambda}\right) \\
& \left.-\frac{5}{4}\left[\sum_{i} L\left(x_{i}^{\Lambda}\right)+\sum_{j} L\left(x_{j}^{\Lambda}\right)+\sum_{k} L\left(x_{k}^{\Lambda+1}\right)+\sum_{l} L\left(x_{l}^{\Lambda+1}\right)\right]-1\right\}
\end{aligned}
$$

where $L(\varphi)=\varphi \ln (\varphi)-\varphi, i, j$ refer to atoms in the layer $\Lambda$, and $k, l$ to atoms in the layer $\Lambda+1$. The expressions (9) and (10), together with the definition $N_{i}=N \sum_{\Lambda} x_{i}^{\Lambda}$, completely specifies the grand potential given in (2).

A simpler expression for the entropy may be obtained within mean-field theory where one considers only the one-site contribution and finds the well-known result

$$
S=-k_{B} N \sum_{\Lambda} c_{\Lambda} \ln c_{\Lambda}+\left(1-c_{\Lambda}\right) \ln \left(1-c_{\Lambda}\right) .
$$

For comparison we have used both expressions for the entropy in the segregation calculations.

The equilibrium concentration-profile may now be determined by minimizing the grand potential $\Omega$ with respect to the four-body variables $z_{i j k l}^{\Lambda}$ and maintaining continuity and normalization by means of the Lagrange multipliers $\alpha_{\Lambda}(i, j)$ and $\lambda_{\Lambda}$, respectively. The minimization produces the following set of equations: 


$$
\begin{aligned}
z_{i j k l}^{(1)}= & \exp \left[\beta \lambda_{1} / 2+\alpha_{1}(i, j)\right] \\
& \times \exp \left\{-\beta \varepsilon_{i j k l}^{(1)}+1 / 8 \beta\left[\mu_{i}+\mu_{j}+\mu_{k}+\mu_{l}\right]\right\} \\
& \times\left[y_{k l}^{(2)} v_{i k}^{(1)} v_{j l}^{(1)} v_{i l}^{(1)} v_{j k}^{(1)}\right]^{1 / 2} \\
& \times\left[x_{i}^{(1)} x_{j}^{(1)}\right]^{1 / 4}\left[x_{l}^{(2)} x_{k}^{(2)}\right]^{-5 / 8} \\
z_{i j k l}^{\Lambda}= & \exp \left[\beta \lambda_{\Lambda} / 2+\alpha_{\Lambda}(i, j)\right] \\
& \times \exp \left\{-\beta \varepsilon_{i j k l}^{\Lambda}+1 / 8 \beta\left[\mu_{i}+\mu_{j}+\mu_{k}+\mu_{l}\right]\right\} \\
& \times\left[y_{i j}^{\Lambda} y_{k l}^{\Lambda+1} v_{i k}^{\Lambda} v_{j l}^{\Lambda} v_{i l}^{\Lambda} v_{j k}^{\Lambda}\right]^{1 / 2} \\
& \times\left[x_{i}^{\Lambda} x_{j}^{\Lambda} x_{l}^{\Lambda+1} x_{k}^{\Lambda+1}\right]^{-5 / 8}
\end{aligned}
$$

which determine the dependence of the multisite correlations on the chemical potentials, interaction parameters, and the temperature $T=1 / k_{B} \beta$. The equations defined by $1 \leq \Lambda \leq M$ must be solved iteratively together with (6).

In the above equations we have included only nearestneighbor interactions. However, it may be necessary to include also the effects of next-nearest neighbors. In traditional CVM these are accounted for by the higher-order clusters, e.g., in the form of the tetrahedron-octahedron approximation. ${ }^{37}$ Unfortunately, one then also has to introduce further interactions defined, for instance, by irregular tetrahedra and isosceles triangles and as a result the number of interactions increases appreciably. For an inhomogeneous system the number of interactions is already rather large and the tetrahedron-octahedron approximation would make the calculations even more complicated. Therefore, in the present case we shall make use of a less accurate but more efficient approximation.

We shall now show how the CVM may be modified in the spirit of the so-called cluster field method ${ }^{38,39}$ (CFM) in order to account for the next-nearest-neighbor interactions between the first (surface) layer and the third layer of the fcc (001) surface. For this purpose we introduce the effective pair interaction $U_{13}$, which depends only on the composition of the first and the third layers. Since any tetrahedron and any pair cluster of atoms in these layers can have only one point in common, we are able to consider their contribution to the statistical sum as independent. Thus, when we solve Eqs. (12) by the natural iteration method, ${ }^{35}$ we may include the $U_{13}$ interaction in the form of the following contributions to the chemical potentials of the species in the first and third layers:

$$
\begin{aligned}
\delta \mu_{i}^{(1)}=(-1)^{i+1} T[ & \ln A+\varepsilon_{13}\left(c_{1}-c_{3}\right) /\left(2 \varepsilon_{13} c_{1}\right) \\
& +U_{13} \varepsilon_{13} y_{1} y_{3} \frac{\partial U_{13}}{\partial c_{1}} \frac{1}{T}\left(1+y_{1}+y_{3}\right) / z_{\mu}^{2} \\
& \left.+\varepsilon_{13} y_{1} y_{3} \frac{\partial U_{13}}{\partial c_{1}} / z_{\mu}\right]
\end{aligned}
$$

$$
\begin{aligned}
\delta \mu_{i}^{(3)}=(-1)^{i+1} T[ & \ln A+\varepsilon_{13}\left(c_{3}-c_{1}\right) /\left(2 \varepsilon_{13} c_{3}\right) \\
& +U_{13} \varepsilon_{13} y_{1} y_{3} \frac{\partial U_{13}}{\partial c_{3}} \frac{1}{T}\left(1+y_{1}+y_{3}\right) / z_{\mu}^{2} \\
& \left.+\varepsilon_{13} y_{1} y_{3} \frac{\partial U_{13}}{\partial c_{3}} / z_{\mu}\right]
\end{aligned}
$$

where

$$
\begin{gathered}
A=c_{1}+c_{3}-1+\left[1+2\left(\varepsilon_{13}-1\right)\left(c_{1}+c_{3}-2 c_{1} c_{3}\right)\right. \\
\left.+\left(c_{3}-c_{1}\right)^{2}\left(\varepsilon_{13}-1\right)^{2}\right]^{1 / 2}, \\
y_{1}=A+\varepsilon_{13}\left(c_{3}-c_{1}\right) /\left[2 \varepsilon_{13}\left(1-c_{3}\right)\right], \\
y_{2}=A+\varepsilon_{13}\left(c_{1}-c_{3}\right) /\left[2 \varepsilon_{13}\left(1-c_{1}\right)\right], \\
\varepsilon_{13}=\exp \left(U_{13} / k T\right), \\
z_{\mu}=1+y_{1}+y_{3}+y_{1} y_{3} \varepsilon_{13},
\end{gathered}
$$

$c_{\Lambda} \equiv\left\langle x_{1}^{\Lambda}\right\rangle$ denotes the concentration of the first component in layer $\Lambda$ and \langle\rangle is the Gibbs average over all possible configurations. The additional terms (13) and (14) derived within the framework of the CFM incorporate only pair contribution since the point term is already included in the CVM result.

\section{CONNOLLY-WILLIAMS AND KIKUCHI INTERACTIONS FOR fcc (001) SURFACES}

In Sec. III we have used the Kikuchi interaction parameters in order to formulate the CVM for an fcc (001) surface. However, in the traditional formulation of the Connolly-Williams method one defines a different and physically more transparent set of parameters. In the present section we shall give the relations between the Connolly-Williams and the Kikuchi interaction parameters.

Let the arrangements of atoms in the semi-infinite fcc crystal be specified by spin variables $\sigma_{\Lambda p}$, which take on the values +1 or -1 depending on the type of atom occupying site $p$ in the layer $\Lambda$. In order to construct the corresponding Hamiltonian we note that there is only one kind of tetrahedra which connects the layers $\Lambda$ and $\Lambda+1$ and that this tetrahedron has two points in each of the layers. As illustrated in Fig. 1 one may therefore define six different kinds of interaction parameters which are (1) $V_{\Lambda}^{(1)}$, the one-site interaction in plane $\Lambda ;(2) V_{\Lambda}^{(2)}$, the two-site interaction in plane $\Lambda$; (3) $V_{\Lambda_{+}}^{(2)}$, the two-site interaction between atoms in the $\Lambda$ and $\Lambda+1$ planes; (4) $V_{\Lambda_{+}}^{(3)}$, the three-site interaction of two atoms in plane $\Lambda$ and an atom in plane $\Lambda+1 ;(5) V_{\Lambda_{++}}^{(3)}$, the three-site interaction of two atoms in plane $\Lambda+1$ and an atom in plane $\Lambda$; and (6) $V_{\Lambda}^{(4)}$, the four-site interaction of atoms in the tetrahedron between the $\Lambda$ and $\Lambda+1$ planes.

Similar to the actual first-principles calculations, one may only treat a finite part of the system and the phenomenological expression for the total energy is therefore best expressed in the form

$$
E=E_{0}+\sum_{\Lambda=1}^{M} E_{\Lambda}^{\mathrm{CWM}}
$$

where $E_{0}$ is a free term which may be neglected in conventional bulk calculations but becomes important for finite systems and $E_{\Lambda}$ is the contribution from layer $\Lambda$ to the energy of the system. $E_{\Lambda}^{C W M}$ for a random alloy may now be defined in terms of the interaction parameters 


$$
\begin{aligned}
E_{\Lambda}^{\mathrm{CWM}}= & V_{\Lambda}^{(1)} \sigma_{\Lambda}+V_{\Lambda}^{(2)} \sigma_{\Lambda}^{2}+\frac{1}{2} V_{\Lambda-1_{+}}^{(2)} \sigma_{\Lambda-1} \sigma_{\Lambda}+\frac{1}{2} V_{\Lambda+1_{+}}^{(2)} \sigma_{\Lambda} \sigma_{\Lambda+1}+\frac{1}{3} V_{\Lambda-1_{+}}^{(3)} \sigma_{\Lambda-1}^{2} \sigma_{\Lambda} \\
& +\frac{2}{3} V_{\Lambda-1_{+}+}^{(3)} \sigma_{\Lambda-1} \sigma_{\Lambda}^{2}+\frac{2}{3} V_{\Lambda_{+}}^{(3)} \sigma_{\Lambda}^{2} \sigma_{\Lambda+1}+\frac{1}{3} V_{\Lambda_{++}}^{(3)} \sigma_{\Lambda} \sigma_{\Lambda+1}^{2}+\frac{1}{2} V_{\Lambda-1}^{(4)} \sigma_{\Lambda-1}^{2} \sigma_{\Lambda}^{2}+\frac{1}{2} V_{\Lambda}^{(4)} \sigma_{\Lambda}^{2} \sigma_{\Lambda+1}^{2}
\end{aligned}
$$

where $\sigma_{\Lambda} \equiv\left\langle\sigma_{\Lambda p}\right\rangle=2 c_{\Lambda}-1$ and for $\Lambda=1$ the terms of the form $V_{\Lambda-1}^{(k)}$ must be excluded. The prefactor of each term in (16) reflects double counting and a particular partition of the totale energy into layer-dependent contributions. Of course, the choice of partition is not unique, especially for three-site interactions, and may only be justified by experience.

The expression for the total energy in terms of the Kikuchi variables has the form equivalent to (15)

$$
E=E_{0}+\sum_{\Lambda=1}^{M} E_{\Lambda}^{K},
$$

where

$$
E_{\Lambda}^{K}=2 \sum_{i j k l} \varepsilon_{i j k l}^{\Lambda} z_{i j k l}^{\Lambda}
$$

It should be noted that $E_{\Lambda}^{K} \neq E_{\Lambda}^{\mathrm{CWM}}$ because $E_{\Lambda}^{K}$ only includes contributions from the tetrahedra that connect the layers $\Lambda$ and $\Lambda+1$. The relation between $\varepsilon_{i j k l}^{\Lambda}$ and $V_{\Lambda}^{(k)}$ may be found by expressing $z_{i j k l}^{\Lambda}$ in terms of $\sigma_{\Lambda p}$, i.e.,

$$
\begin{aligned}
z_{i j k l}^{\Lambda}=\frac{1}{16} & \left\langle\left[1+(-1)^{i+1} \sigma_{\Lambda p}\right]\left[1+(-1)^{j+1} \sigma_{\Lambda q}\right]\right. \\
& \left.\times\left[1+(-1)^{k+1} \sigma_{\Lambda r}\right]\left[1+(-1)^{l+1} \sigma_{\Lambda s}\right]\right\rangle
\end{aligned}
$$

When we note that $i, j, k$, and $l$ designate types of atoms and can only take on the values 1 or 2 we obtain the following expressions for $\varepsilon_{i j k l}^{\Lambda}$ :

$$
\begin{aligned}
& \varepsilon_{1111}^{\Lambda}=\frac{1}{2}\left(V_{\Lambda}^{(1)}+V_{\Lambda+1}^{(1)}+V_{\Lambda}^{(2)}+V_{\Lambda_{+}}^{(2)}+V_{\Lambda+1}^{(2)}\right. \\
& \left.+V_{\Lambda_{+}}^{(3)}+V_{\Lambda_{++}}^{(3)}+V_{\Lambda}^{(4)}\right) \\
& \varepsilon_{1112}^{\Lambda}=\varepsilon_{1121}^{\Lambda}=\frac{1}{2}\left(V_{\Lambda}^{(1)}+V_{\Lambda}^{(2)}-V_{\Lambda+1}^{(2)}-V_{\Lambda_{++}}^{(3)}-V_{\Lambda}^{(4)}\right), \\
& \varepsilon_{1122}^{\Lambda}=\frac{1}{2}\left(V_{\Lambda}^{(1)}-V_{\Lambda+1}^{(1)}+V_{\Lambda}^{(2)}-V_{\Lambda+1}^{(2)}\right. \\
& \left.+V_{\Lambda_{+}}^{(2)}-V_{\Lambda_{+}}^{(3)}+V_{\Lambda_{+}}^{(3)}+V_{\Lambda}^{(4)}\right) \\
& \varepsilon_{1211}^{\Lambda}=\varepsilon_{2111}^{\Lambda}=\frac{1}{2}\left(V_{\Lambda+1}^{(1)}-V_{\Lambda}^{(2)}+V_{\Lambda+1}^{(2)}-V_{\Lambda_{+}}^{(3)}-V_{\Lambda}^{(4)}\right), \\
& \varepsilon_{1212}^{\Lambda}=\varepsilon_{1221}^{\Lambda}=\varepsilon_{2112}^{\Lambda}=\varepsilon_{2121}^{\Lambda} \\
& =\frac{1}{2}\left(-V_{\Lambda+1}^{(2)}-V_{\Lambda}^{(2)}+V_{\Lambda}^{(4)}\right),
\end{aligned}
$$

$$
\begin{aligned}
\varepsilon_{1222}^{\Lambda}=\varepsilon_{2122}^{\Lambda}=\frac{1}{2}( & -V_{\Lambda+1}^{(1)}-V_{\Lambda}^{(2)}+V_{\Lambda+1}^{(2)} \\
& \left.+V_{\Lambda_{+}}^{(3)}-V_{\Lambda}^{(4)}\right) \\
\varepsilon_{2211}^{\Lambda}=\frac{1}{2}( & -V_{\Lambda}^{(1)}+V_{\Lambda+1}^{(1)}+V_{\Lambda}^{(2)}-V_{\Lambda_{+}}^{(2)} \\
& \left.+V_{\Lambda+1}^{(2)}+V_{\Lambda_{+}}^{(3)}-V_{\Lambda_{+}}^{(3)}+V_{\Lambda}^{(4)}\right),
\end{aligned}
$$$$
\varepsilon_{2222}^{\Lambda}=\frac{1}{2}\left(-V_{\Lambda}^{(1)}-V_{\Lambda+1}^{(1)}+V_{\Lambda}^{(2)}+V_{\Lambda_{+}}^{(2)}\right.
$$$$
\left.+V_{\Lambda+1}^{(2)}-V_{\Lambda_{+}}^{(3)}-V_{\Lambda_{++}}^{(3)}+V_{\Lambda}^{(4)}\right) \text {, }
$$

$$
\begin{aligned}
\varepsilon_{2212}^{\Lambda}=\varepsilon_{2221}^{\Lambda}=\frac{1}{2}( & -V_{\Lambda}^{(1)}+V_{\Lambda}^{(2)}-V_{\Lambda+1}^{(2)} \\
& \left.+V_{\Lambda_{++}}^{(3)}-V_{\Lambda}^{(4)}\right) .
\end{aligned}
$$

\section{DETAILS OF CALCULATIONS}

The analysis of the short-range-order data for $\mathrm{Cu}-\mathrm{Ni}$ alloys ${ }^{40}$ as well as the effective pair interaction calculations ${ }^{17,40}$ indicate (1) that the effective pair interactions in these alloys depend strongly on the concentration and (2) that the values of the first- and secondneighbor pair interactions are practically equal and much greater than all the other more distant interactions. The first fact means that the concentration independent Connolly-Williams three- and four-site interactions must be comparable to the two-site interactions. The second fact shows that the tetrahedron approximation is insufficient to determine the configurational properties of $\mathrm{Cu}-\mathrm{Ni}$ alloys.

In the present work we still apply the tetrahedron approximation to the semi-infinite system but add to it the contribution from the interaction between the surface and the third layer as described in Sec. III. With these approximations we obviously cannot obtain the correct equilibrium short-range atomic configuration in the $\mathrm{Cu}-\mathrm{Ni}$ alloys. However, usually short-range-order effects only slightly influence the thermodynamic properties. Besides, the main aim of our calculations is the determination of equilibrium surface concentration profiles, and for the $\mathrm{Cu}-\mathrm{Ni}$ system as well as for most systems only the first two layers exhibit a significant deviation from the bulk concentration.

To obtain the multisite interactions we have performed total-energy calculations for a series of random (001) surfaces of $\mathrm{Cu}-\mathrm{Ni}$ alloys by means of the LMTO-CPA technique described in detail in Ref. 27. In these calculations we treated the four outermost layers self-consistently as- 
TABLE II. The configurations used in the self-consistent LMTO-CPA calculations in order to determine the $17 \mathrm{CVM}$ and the additional 4 CFM interatomic interaction parameters. The concentration within the bulk and within all other layers were equal to the concentration of the third layer.

\begin{tabular}{llllllllllllllllllllll}
\hline \hline$\Lambda$ & 110 \\
\hline 1 & 50 & 88 & 12 & 50 & 50 & 50 & 50 & 50 & 50 & 12 & 88 & 12 & 88 & 50 & 50 & 50 & 50 & 12 & 88 & 88 & 12 \\
2 & 50 & 50 & 50 & 12 & 88 & 50 & 50 & 50 & 50 & 88 & 12 & 12 & 88 & 12 & 88 & 12 & 88 & 50 & 50 & 50 & 50 \\
3 & 50 & 50 & 50 & 50 & 50 & 12 & 88 & 25 & 75 & 88 & 12 & 12 & 88 & 88 & 12 & 12 & 88 & 12 & 12 & 88 & 88 \\
\hline \hline
\end{tabular}

suming the concentration profiles specified in Table II. We then proceeded to evaluate the CVM interaction parameters by inverting the expansion (15) carried to $M=4$ under the simplifying assumption that fourth layer may be considered to be a bulk layer whereby

$$
V_{3}^{(2)}=\frac{1}{2} V_{3_{+}}^{(2)}, \quad V_{3_{+}}^{(3)}=V_{3_{+}}^{(3)} .
$$

All other interactions beginning from the fourth layer are per definition equal to their bulk values which were evaluated from LMTO-CPA bulk calculations for $c_{\mathrm{Cu}}=$ $88 \%, 75 \%, 50 \%, 25 \%$, and $12 \%$. With the above assumptions the total number of different CVM interactionparameters is 17 . However, in order to include the $U_{13}$ interaction between the first and the third layers we need the four additional parameters $u^{(k)}$ which enter the CFM expression

$U_{13}=u^{(2)} \sigma_{1} \sigma_{3}+u_{+}^{(3)} \sigma_{1}^{2} \sigma_{3}+u_{++}^{(3)} \sigma_{1} \sigma_{3}^{2}+u^{(4)} \sigma_{1}^{2} \sigma_{3}^{2}$,

where $\sigma_{1}$ and $\sigma_{3}$ are the averaged Ising spin variables for the first and the third layers, respectively. When (22) is added to the expansion (15) there are a total number of 21 interatomic interaction parameters to be determined.

We have tested the accuracy of the determination of the Connolly-Williams interactions by performing totalenergy calculations for a number of additional choices of concentration profile including sets with $c_{\mathrm{Cu}}^{\max }=100 \%$ and $c_{\mathrm{Cu}}^{\min }=4 \%$. In all cases the difference between the interactions obtained were found to be similar to the convergence of LMTO-CPA total-energy calculations, i.e., $\sim 0.001 \mathrm{mRy}$. In addition, on the basis of the ConnollyWilliams parameters we repeated the surface-energy calculations to be presented later in Fig. 5 and found that the CWM expansion gave results which were indistinguishable from the LMTO-CPA results.

We should also mention that in the evaluation of the Connolly-Williams interaction parameters by means of (15) we have not partitioned the LMTO-CPA total energy into its layer contributions, although this is possible within the atomic sphere approximation. This is important because such a partition of the total energy is not unique and, if it was carried out, one would not treat the vacuum layers correctly.

Finally, we have found that the interaction parameters in the $\mathrm{Cu}-\mathrm{Ni}$ system are essentially independent of volume in the range specified by pure $\mathrm{Cu}$ and $\mathrm{Ni}$. Therefore, we have calculated the so-called globally relaxed interactions ${ }^{41}$ rather than the locally relaxed interactions. The Wigner-Seits radius of all the CWM calcula- tions was equal to 2.614 bohrs, which corresponds to the calculated equilibrium bulk lattice spacing of a $\mathrm{Cu}_{50} \mathrm{Ni}_{50}$ alloy. ${ }^{27}$

\section{ELECTRONIC STRUCTURE OF (001) SURFACE FOR RANDOM Cu-Ni ALLOYS}

In this section we present the calculated electronic densities of states (DOS) for the (001) surface of random $\mathrm{Cu}-\mathrm{Ni}$ alloys. This system has recently been investigated by Kudrnovsky et al. ${ }^{42}$ Here we will focus the attention on the surface electronic structure as a function of surface concentration and on the comparison with the experimental spectra obtained by ultraviolet-photoelectron spectroscopy (UPS) by Yu et al. ${ }^{43}$

\section{A. (001) surface of the uniformly random $\mathrm{Cu}_{50} \mathrm{Ni}_{50}$ alloy}

In Fig. 2 we present the results for a uniformly random $\mathrm{Cu}_{50} \mathrm{Ni}_{50}$ alloy as a function of distance from the surface where by uniformly random we mean that the concentrations in all the atomic layers, including the surface layer, are chosen to be the same as in the bulk. The results show excellent agreement with the surface state densities recently reported by Kudrnovsky et al. ${ }^{42}$ for the same alloy composition. The bulk electronic structure for this system has also been studied previously by means of the CPA (Refs. 44 and 45) and our state density results for the third layer of the semi-infinite crystal agree well with these earlier bulk calculations. The bulk DOS is dominated by two peaks which corresponds to the $\mathrm{Cu} d$ band $(-0.4$ to -0.1 Ry below the Fermi energy) and Ni $d$ band $(-0.3$ to $0.0 \mathrm{Ry})$. As would be expected for a strongly disordered system there is no detailed fine structure. Even when we go to the surface layer, this general structure of the DOS does not change and the spectrum still consists of two structureless peaks related to the $d$ bands of $\mathrm{Cu}$ $(-0.3$ to $-0.05 \mathrm{Ry})$ and $\mathrm{Ni}(-0.2$ to $0.05 \mathrm{Ry})$.

The band narrowing effect at the surface is clearly seen both in the total DOS and in the DOS projected onto the $\mathrm{Cu}$ and $\mathrm{Ni}$ atoms separately. The effect is due to the lower coordination number of the surface atoms. In a simple nearest-neighbor tight-binding model the bandwidth is proportional to the square root of the coordination number ${ }^{46}$ and a reduction of the width of about $\sqrt{8 / 12}$ may therefore be expected on the (001) surface. This is in fact rather close to what is obtained in the calculation. 
The band narrowing at a surface leads to a shift in the center of gravity of the $d$ band. If the number of $d$ electrons $n_{d}$ at the surface remains close to the bulk value, the shift of the correspondent $d$ band center for the $\mathrm{Cu}$ or $\mathrm{Ni}$ band may be estimated within the rectangular band model $^{47}$ to be $0.02 W_{j}\left(n_{d}-5\right)$, where $W_{j}$ is a bulk band width for the $j$ th alloy component. Thus, for metals with a more than half filled $d$ band the shift is positive, in agreement with our results for $\mathrm{Cu}$ and $\mathrm{Ni}$. A more quantitative estimate may be provided by the LMTO band center parameter $C$. We find that $C^{\text {surf }}-C^{\text {bulk }}$ is 32 $\mathrm{mRy}$ for $\mathrm{Cu} d$ band and $27 \mathrm{mRy}$ for $\mathrm{Ni} d$ band. As a result the alloy $d$ band is also shifted towards the higher energies.

It is also seen that the DOS is almost completely bulklike already in the first subsurface layer due to an efficient screening. Outside the surface the DOS exhibits two peaks coming from the $\mathrm{Cu}$ and $\mathrm{Ni} d$ states reaching out into the vacuum region, where they, when decomposed in terms of partial waves in the atomic sphere outside the surface, show up mainly in the $s$-projected DOS.

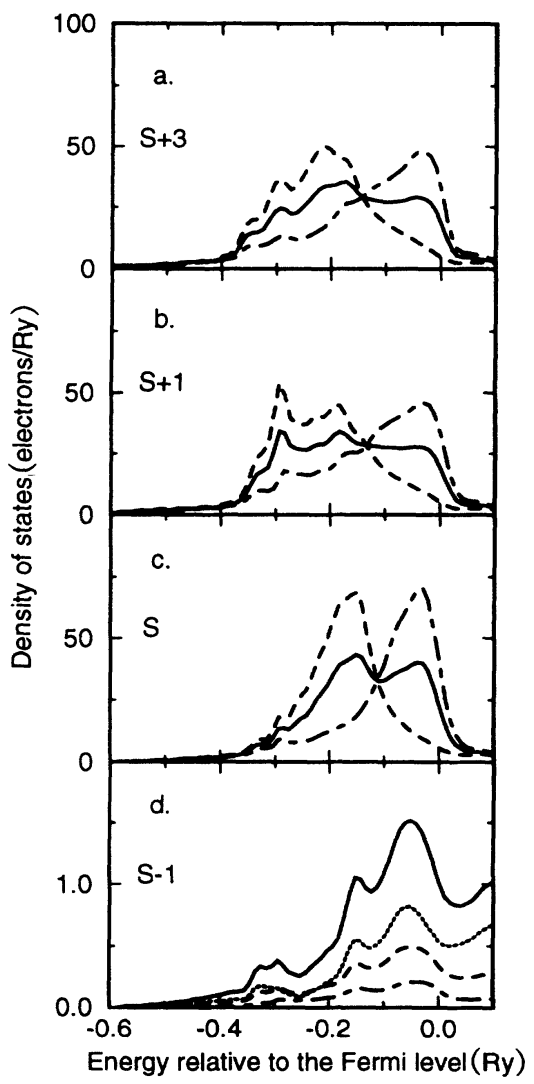

FIG. 2. Density of states for the (001) surface of a uniform $\mathrm{Cu}_{50} \mathrm{Ni}_{50}$ alloy. In (a)-(c) shown is the local DOS projected onto the atomic sphere of the $S+i$ th layer, where $S$ is the surface layer and a positive $i$ designates the alloy region. The total DOS is shown by a full line, the local Cu DOS by a dashed line, and the local Ni DOS by a dot-dashed line. In (d) the local DOS is shown projected onto the vacuum atomic sphere in the $S-1$ st layer. The total DOS is shown by a full line, the partial $s$ DOS by a dotted line, the partial $p$ DOS by a dashed line, and the partial $d$ DOS by a dot-dashed line.
Note that the $\mathrm{Ni}$ states clearly dominate. This is due to the fact that the $\mathrm{Ni} d$ states lie higher in energy than those of $\mathrm{Cu}$ whereby the decay length for the $\mathrm{Ni}$ wave functions into the vacuum region is longer.

In conclusion, it is seen that the electronic structure when going from the bulk region to the surface of a uniformly disordered $\mathrm{Cu}-\mathrm{Ni}$ alloys exibits the same general trends as pure $\mathrm{Cu}$ and $\mathrm{Ni} .{ }^{48-51}$ However, the presence of surface segregation may radically change this situation, as we shall discuss in the following section.

\section{B. (001) surfaces of segregated $\mathrm{Cu}-\mathrm{Ni}$ alloys}

The results for the alloys with bulk $\mathrm{Cu}$ concentrations of $50 \%$ and $10 \%$ and with different surface compositions are presented in Figs. 3 and 4, respectively. We choose these alloys in order to compare with UPS spectra. ${ }^{43}$ In

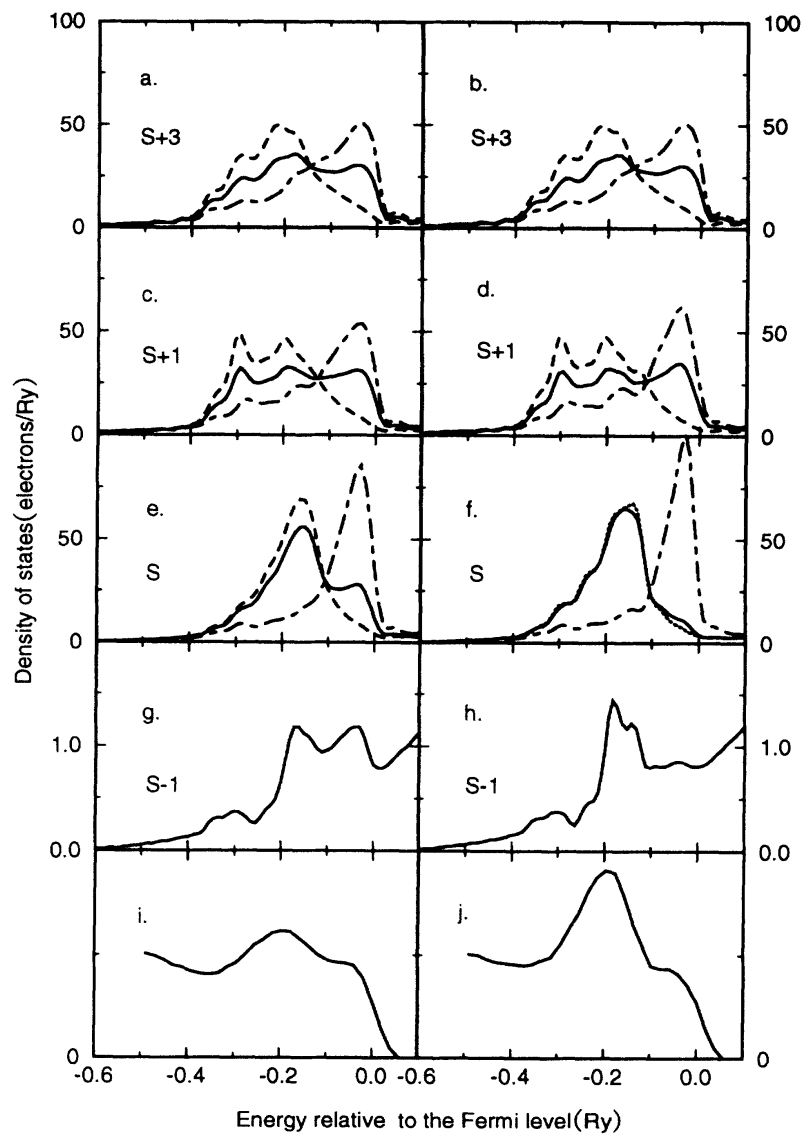

FIG. 3. Density of states for (001) surface of a $\mathrm{Cu}_{50} \mathrm{Ni}_{50}$ alloy with a surface concentration of $75 \% \mathrm{Cu}$ (left panel) and $96 \%$ (right panel). In (a)-(f) the local DOS projected onto the atomic sphere of $S+i$ th layer is shown (see caption of Fig. 2). The total DOS is shown by a full line, the local DOS for Cu by a dashed line, and the local DOS for Ni by a dot-dashed line. The dotted line in (f) denotes the surface DOS for a $\mathrm{Cu}$ overlayer. In (g) and (h) the local DOS projected onto the vacuum atomic sphere of the $S-1$ st layer is shown. In (i) and $(\mathrm{j})$ are shown the experimental UPS spectra in relative units from Ref. 43. 


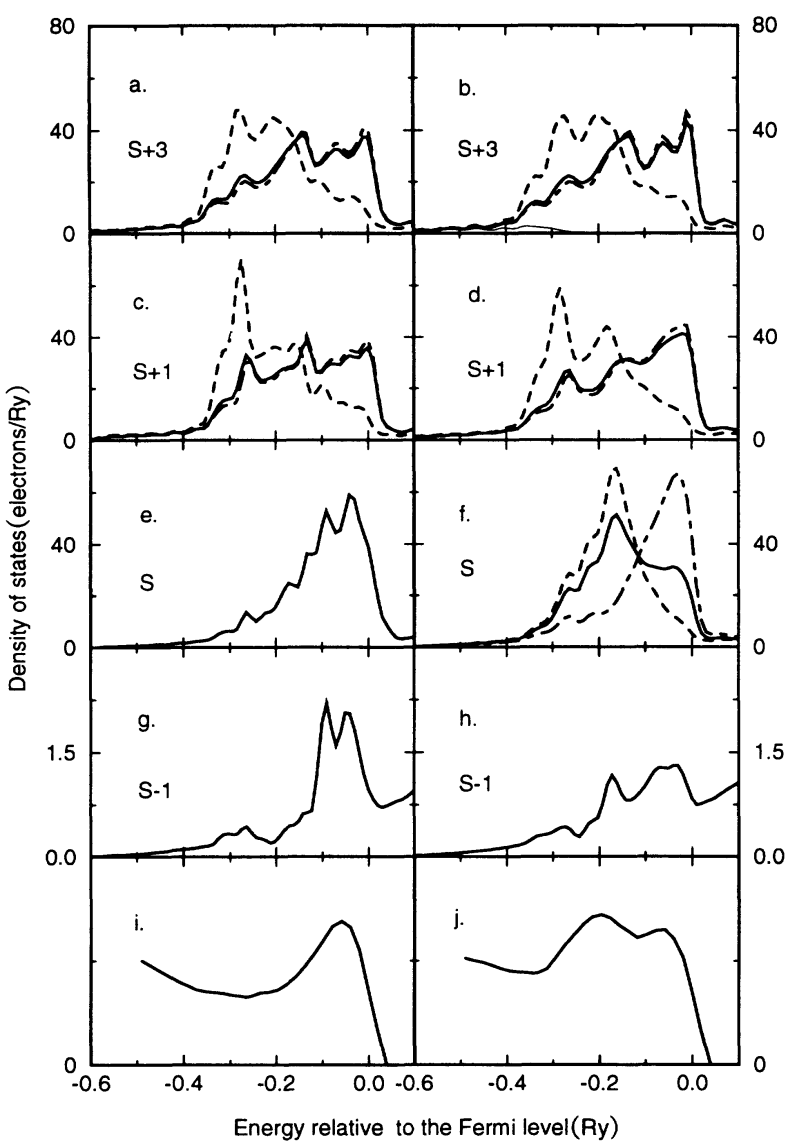

FIG. 4. Density of states for the (001) surface of a $\mathrm{Cu}_{10} \mathrm{Ni}_{90}$ alloy with a surface concentration of $100 \% \mathrm{Ni}$ (left panel) and $35 \% \mathrm{Ni}$ (right panel). The notation is the same as in Fig. 3.

this comparison we neglect the effects of matrix elements and inelastic scattering but still expect the main features of the electronic state density to be reflected in the UPS spectrum.

As one may see from the figures, the agreement between the calculated densities of states at the surface and the UPS spectra is indeed quite good [panels (e) and (i), and (f) and (j) on Figs. 3 and 4]. The general structure of the spectra both with respect to the positions of the peaks and their changes in relative intensities with surface composition is reproduced in the calculations. This agreement may be viewed as a measure of the applicability of the single-site CPA to the case of surfaces of random $\mathrm{Cu}-\mathrm{Ni}$ alloys.

A comparison of Figs. 2(c), 3(e), and 3(f) shows that as the surface $\mathrm{Cu}$ concentration increases the intensity of the $\mathrm{Cu}$-related peak in the total density of states at the surface increases at the expense of the Ni-related state density. This is a simple dilution effect. The sharp peak in the local density of states at the $\mathrm{Ni}$ atom is narrowed and for a $\mathrm{Cu}$ surface concentration of $96 \%$ it has the character of a virtually bound state at the energy -0.03 Ry. This narrow resonance was also found in dilute $\mathrm{Cu}$ rich alloys, ${ }^{52}$ and one consequence of its appearance is that, as in the bulk case, one cannot use the rigid band model to describe the surface electronic structure for $\mathrm{Cu}-\mathrm{Ni}$ alloys.
The narrow resonance at the $\mathrm{Ni}$ atoms appears as a shoulder in the total density of states in the high energy region and such a shoulder is indeed observed in the UPS spectra ${ }^{43}$ of an annealed alloy with a bulk $\mathrm{Cu}$ concentration of $50 \%$. If we add a pure $\mathrm{Cu}$ overlayer to the $\mathrm{Cu}_{50} \mathrm{Ni}_{50}$ uniform alloy the shoulder disappears as indicated by the dashed curve in Fig. 3(f). Hence the existence of this shoulder in the UPS spectrum for the annealed sample indicates the presence of $\mathrm{Ni}$ atoms at the surface. This in agreement with the segregation calculations to be presented in the next section which indicates a small but finite concentration of $\mathrm{Ni}$ at the surface.

As for the Cu-related peak, it becomes a little wider at the surface as the $\mathrm{Cu}$ concentration increases, and some fine structure begins to appear. This last effect is a consequence of the increased probability for a $\mathrm{Cu}$ surface atom to be surrounded by mostly $\mathrm{Cu}$ neighbors. However, still the fine structure is not nearly as pronounced as in a bulk Cu-rich alloy ${ }^{52}$ because of the much higher concentration of $\mathrm{Ni}$ atoms in the subsurface layer.

Analogous changes in the electronic structure can be seen for the alloy with a bulk $\mathrm{Cu}$ concentration of $10 \%$ as the surface concentration of $\mathrm{Cu}$ increases. The DOS at the surface for the nonequilibrium alloy with a pure $\mathrm{Ni}$ surface is of course dominated by the contribution from the $d$ electrons of $\mathrm{Ni}$ and the spectrum exhibits a well developed fine structure similar to the one on a pure $\mathrm{Ni}$ crystal. ${ }^{49-51}$ As the surface concentration of $\mathrm{Cu}$ increases up to $65 \%$, the Ni DOS loses its fine structure and the contribution from the $\mathrm{Cu} d$ band appears in the total DOS in complete agreement with the UPS data. ${ }^{43}$

The density of states outside the surface, projected onto the first atomic sphere above the surface, undergoes changes very similar to those of the surface layer. Note, however, that the relative intensities of the $\mathrm{Cu}$ and Ni-related peaks are not proportional to the surface composition due to the fact that the Ni $d$ states penetrate further into the vacuum region. Again, the screening of the surface layer is seen to be very efficient as we go into the bulk of the crystal. Indeed, the electronic structure in the third layer below the surface appears to be completely independent of the surface composition. Even the first subsurface layer is only slightly affected, although one may see in Figs. 2(b), 3(c), and 3(d) that the Ni-related peak gets more narrow and its intensity is increased slightly as the surface concentration of $\mathrm{Cu}$ is increased for the $\mathrm{Cu}_{50} \mathrm{Ni}_{50}$ alloy. It should noted that in these calculations we change only the surface composition which will only affect 4 of the 12 nearest neighbors of an atom in the first subsurface layer.

One may conclude that surface segregation will be important for the electronic structure of random alloy surfaces. At the same time, the agreement between the calculated density of states and the experimental UPS spectra indicates that the CPA may be used to describe surface properties of random $\mathrm{Cu}-\mathrm{Ni}$ alloys with good accuracy.

\section{WORK FUNCTION}

In Table III we present the work function calculated as a function of the surface concentration and compare it to 
TABLE III. Work function $\phi$ of an fcc (001) surface of $\mathrm{Cu}-\mathrm{Ni}$ alloys for different concentrations.

\begin{tabular}{cccc}
\hline \multicolumn{2}{c}{$\begin{array}{c}\text { Concentration of } \mathrm{Cu} \text { in at. \% } \\
\text { Bulk }\end{array}$} & \multicolumn{2}{c}{$\phi(\mathrm{eV})$} \\
Surface & Theory & Expt. $^{\text {a }}$ \\
\hline 50 & 100 & 5.3 & 4.6 \\
50 & 75 & 5.4 & 4.7 \\
10 & 65 & 5.5 & 4.6 \\
10 & 0 & 5.8 & 5.0 \\
\hline \hline
\end{tabular}

${ }^{\mathrm{a}}$ Reference 43.

experiments. ${ }^{43}$ As expected, the work function decreases with an increasing surface concentration of $\mathrm{Cu}$, and these changes are reproduced in the calculations with good accuracy. The deviation between the calculated and the experimental absolute values is of the order of $15 \%$, which is typical in the present kind of calculations and may be caused by inperfections at the surface as suggested in Ref. 53.

\section{SURFACE ENERGY FOR $\mathrm{Cu}_{50} \mathrm{Ni}_{50}$ WITH SEGREGATION}

\section{A. Layer dependence}

Before we present the temperature dependent segregation profiles obtained by the CVM method we shall show by direct surface-energy calculations that an oscillatory concentration profile is to be expected. To do this, we consider a $50 \% \mathrm{CuNi}$ alloy and vary only the concentration of the surface layer. The surface energy of this system calculated by (3-5) is shown in Fig. 5(a) from where it is seen that the lowest surface energy is reached when the alloy surface is fully covered by $\mathrm{Cu}$. This means that $\mathrm{Cu}$ will exhibit a strong tendency to segregate towards the surface and is in complete agreement with the notion that the element which has the lowest surface energy will cover the surface of its alloys.

We then fix the concentration of the surface layer at a $100 \%$ and proceed to vary the concentration of the first subsurface layer. The results are presented in Fig. 5(b), and they show that in this case the lowest surface energy is found when the subsurface layer is completely depleted for $\mathrm{Cu}$. We point out that, although the variation of the surface energy with concentration is much smaller for the subsurface layer than for the surface layer, the minimum at zero concentration is well within the numerical accuracy of the LMTO-CPA technique.

Although we have performed only a restricted variation of the surface energy within the space spanned by the concentrations of the two topmost surface layers, the smooth variation of the calculated surface energies strongly indicates that the absolute minimum will be found for $c_{1}=100 \%$ and $c_{2}=0 \%$. Consequently, the segregation profile in $\mathrm{CuNi}$ alloys should be oscillatory rather than monotonic. As we shall see this expectation is borne out by the segregation caculations to be presented in Sec. IX B.

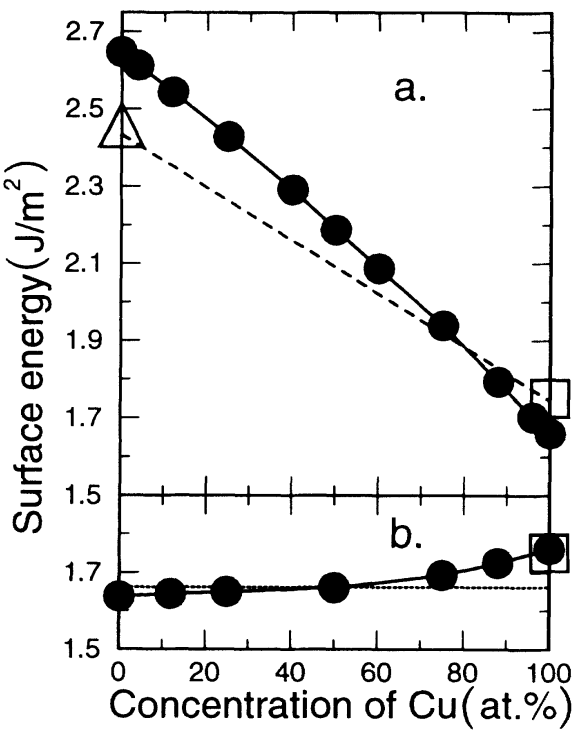

FIG. 5. The surface energy of the (001) surface of a random $\mathrm{Cu}_{50} \mathrm{Ni}_{50}$ alloy as calculated by means of LMTO-CPA (full line, filled circles). In (a) the concentration of the surface layer is changed while the concentration of the subsurface layers is kept at $50 \% \mathrm{Cu}$. In (b) the concentration of the first subsurface layer is changed while the concentration of the surface layer is kept at $100 \%$ and the concentration of the remaining subsurface layers is fixed at $50 \%$. The concentration average (dashed line) of the surface energies for pure $\mathrm{Cu}$ (open square) and $\mathrm{Ni}$ (open triangle) is also shown. The dotted line in (b) represents the minimum surface energy in (a), i.e., the surface energy of the alloy with $100 \% \mathrm{Cu}$ in the surface layer and $50 \% \mathrm{Cu}$ in the subsurface layers.

\section{B. Concentration dependence}

It may seen from Fig. 5(a) that the calculated surface energy depends essentially linearly on the concentration. Now, the mixing enthalpy of the $\mathrm{Cu}-\mathrm{Ni}$ system is found to be small over the whole concentration range ${ }^{27}$ and hence one may assume that the total energy per atom of $\mathrm{Cu}$ and $\mathrm{Ni}$ is independent of alloy concentration. In that case one gets for the chemical potential (4) simply $\mu_{1}=E_{\mathrm{Cu}}^{\text {bulk }}-E_{\mathrm{Ni}}^{\text {bulk }}$ and, if one assumes that the vacuum contribution may be assigned to the surface layer, the surface energy becomes

$E_{\text {surf }}=\sum_{\Lambda=1}^{M}\left[c_{\Lambda}\left(E_{\Lambda}^{\mathrm{Cu}}-E_{\mathrm{Cu}}^{\mathrm{bulk}}\right)+\left(1-c_{\Lambda}\right)\left(E_{\Lambda}^{\mathrm{Ni}}-E_{\mathrm{Ni}}^{\mathrm{bulk}}\right)\right]$

If all subsurface concentrations are fixed at the bulk value of $50 \%,(23)$ leads to a surface energy which is linear in $c_{1}$. The self-consistent result shown in Fig. 5(a) deviates only slightly from this expected linear behavior, the deviation being a measure of the validity of the assumption of concentration independent total energies for $\mathrm{Cu}$ and $\mathrm{Ni}$.

If the concentration of all surface layers is independent of $\Lambda$ and equal to a common value $c$, one obtains from 
(23) a surface energy which varies with $c$ as the concentration average of the surface energies of pure $\mathrm{Ni}$ and $\mathrm{Cu}$. As seen in Fig. 5(a), this is a reasonable approximation for the random overlayer on $\mathrm{Cu}_{50} \mathrm{Ni}_{50}$. Here the deviation from the concentration average is a measure of the effect of alloying and the fact that the self-consistent result falls below the concentration average for $c>80 \%$ means that the tendency for $\mathrm{Cu}$ to segregate towards the surface is enhanced by alloying.

In the case where only the concentration of the subsurface layer is changed [Fig. 5(b)] the variation of the surface energy deviates somewhat from the expected linear behavior, but still the variation is monotonic. In addition the variation with concentration is quite weak, reflecting the fact that the main contribution to the surface energy in the ASA partition comes from the surface layer and the first vacuum layer. This is in agreement with the observation that the state density of the first subsurface layer shown in Sec. VI is close to the bulk result and hence the differences $E_{2}^{\mathrm{Cu}}-E_{\text {bulk }}^{\mathrm{Cu}}$ and $E_{2}^{\mathrm{Ni}}-E_{\text {bulk }}^{\mathrm{Ni}}$ are much smaller than the corresponding values for $\Lambda=1$.

Finally, it is interesting to note that the surface energy of the system of two $\mathrm{Cu}$ overlayers on top of the random alloy, $c_{2}=100 \%$ in Fig. $5(\mathrm{~b})$, is very close to the surface energy of pure $\mathrm{Cu}$. This reflects the fact that the $\mathrm{Cu}$ atom on the surface of the alloy has exactly the same environment as on the surface of pure $\mathrm{Cu}$ and that the subsurface $\mathrm{Cu}$ atom in the alloy on the average has only exchanged 2 of its 12 neighbors with $\mathrm{Ni}$.

\section{INTERATOMIC INTERACTIONS AND SEGREGATION PROFILE FOR Cu-Ni ALLOYS}

\section{A. Connolly-Williams parameters}

The Connolly-Williams interactions calculated on the basis of the LMTO-CPA total energies are presented in Table IV and the additional interaction parameters needed in (22) as well as the free parameter in (15) are given in Table V. We notice that essentially all the interaction parameters in Table IV are of the same order of magnitude. This may be understood as follows. Since we treat completely random alloys, we cannot distinguish contributions to the total energy from different shells, i.e., our $V_{\Lambda}^{(2)}$ and $V_{\Lambda_{+}}^{(2)}$ are equal to the sum of all pair interactions in the layer $\Lambda$ or between layers $\Lambda$ and $\Lambda+1$. On the other hand, the effective pair interactions within the first and the second coordination shells of the $\mathrm{Cu}-\mathrm{Ni}$ system are large but of opposite signs. ${ }^{17,40}$ Thus their contributions to the total energy compensate and lead to
TABLE V. Additional interaction parameters used in Eqs. (15) and (22) in degrees $\mathrm{K}$.

\begin{tabular}{lrrrr}
\hline \hline$E_{0}^{\text {surf }}-E_{0}^{\text {bulk }}$ & $u^{(2)}$ & $u_{+}^{(3)}$ & $u_{+ \pm}^{(3)}$ & $u^{(4)}$ \\
\hline 9949.35 & 87.80 & 84.45 & 71.58 & 63.08 \\
\hline \hline
\end{tabular}

a relatively small value for $V_{\Lambda}^{(2)}$. Finally, the relatively large values for the three- and four-site interactions reflect the strong concentration dependence of the effective pair interactions which is also observed in the analysis of the experimental neutron scattering data ${ }^{40}$ and in the calculations. ${ }^{17,40}$

It may be seen in Table IV that the interactions viewed as functions of the layer number $\Lambda$ converge towards their bulk values except for the four-site interactions where $V_{3}^{(4)}$ is relatively large. This is caused by the cutoff at $\Lambda=4$ and the assumptions (21) whereby part of the total energy which is not accounted for by the other interactions gets assigned to $V_{3}^{(4)}$ in the Connolly-Williams procedure. However, the exact value for this four-site interaction has little effect on the calculated segregation profiles.

The contribution to the total energy from the interlayer interactions in Table IV may be discussed in the light of (16). Thus the fact that $V_{1_{+}}^{(2)}$ is negative means that the configurations with the same element in both layers, i.e., either $\left(\sigma_{1}, \sigma_{2}\right)=(1,1) \sim\{\mathrm{Cu}, \mathrm{Cu}, \ldots\}$ or $\left(\sigma_{1}, \sigma_{2}\right)=(-1,-1) \sim\{\mathrm{Ni}, \mathrm{Ni}, \ldots\}$ will be preferred. Furthermore, the positive sign of $V_{1_{+}}^{(3)}$ and $V_{1_{++}}^{(3)}$ favors the compositions $\{\mathrm{Cu}, \mathrm{Ni}, \ldots\}$ and $\{\mathrm{Ni}, \mathrm{Cu}, \ldots\}$, while the negative sign of $V_{1}^{(4)}$ make all the compositions equally favorable. Since the values of $V_{1_{+}}^{(2)}$ and $V_{1_{+}}^{(3)}, V_{1_{+}+}^{(3)}$ are comparable, the equilibrium surface concentration profile will be determined by the large values of $V_{1}^{(1)}$ and $V_{2}^{(1)}$, which favor $\mathrm{Cu}$ in the first layer and $\mathrm{Ni}$ in the second layer. As a result, we may expect a strong segregation of $\mathrm{Cu}$ into the surface layer and a weak segregation of $\mathrm{Ni}$ into the first subsurface layer which of course is a direct consequence of the concentration dependence of the LMTO-CPA surface energies presented in Fig. 5.

It is interesting to note that our values for $V_{1}^{(1)}$ and $V_{3}^{(1)}$ are close to those obtained in the GPM calculations $\sim 1800 \mathrm{~K}$ and $\sim-15 \mathrm{~K}$, respectively, ${ }^{54}$ while $V_{2}^{(1)}$ does not agree with the GPM results. The value for $V_{1}^{(1)}$ obtained in Ref. 16 by the semiempirical tight-binding method is approximately twice our result. However, although the Connolly-Williams method itself has certain deficiencies, it may be more realistic than the GPM, be-

TABLE IV. Connolly-Williams interactions for the $\mathrm{Cu}-\mathrm{Ni}(001)$ surface in degrees $\mathrm{K}$.

\begin{tabular}{lrrrrrr}
\hline \hline$\Lambda$ & $V_{\Lambda}^{(1)}-V_{\text {bulk }}^{(1)}$ & \multicolumn{1}{c}{$V_{\Lambda}^{(2)}$} & \multicolumn{1}{c}{$V_{\Lambda_{+}}^{(2)}$} & \multicolumn{1}{c}{$V_{\Lambda_{+}}^{(3)}$} & \multicolumn{1}{c}{$V_{\Lambda_{+}}^{(3)}$} & \multicolumn{1}{c}{$V_{\Lambda}^{(4)}$} \\
\hline 1 & -2085.54 & -198.12 & -108.97 & 36.30 & 61.27 & -75.89 \\
2 & 398.82 & 75.51 & 6.96 & 36.65 & 108.71 & 62.17 \\
3 & -25.39 & -75.00 & -50.00 & 129.74 & 129.74 & 346.30 \\
Bulk & & -10.11 & -20.21 & 95.82 & 95.82 & 124.12 \\
\hline
\end{tabular}


cause it is based on a series of accurate total-energy calculations.

\section{B. Segregation proflles for the (001) surface}

In Fig. 6 we present the equilibrium concentration profiles of the (001) surface of random $\mathrm{Cu}-\mathrm{Ni}$ alloys for two temperatures and a variety of bulk concentrations as obtained by means of the generalized cluster variation method described in Sec. III. In addition, we include the results of the mean-field expression (11) for the entropy. The difference between the two sets of results is small and this confirms the expectation that short-range order has little effect on the equilibrium composition of an alloy surface at temperatures above the bulk orderdisorder temperature. Unfortunately, since we cannot separate nearest-neighbor from next-nearest-neighbor interactions, it is not possible to confirm that the CVM expression for the entropy is more accurate than mean-field theory.

We have compared the present segregation profiles for two selected bulk concentrations with the results of four

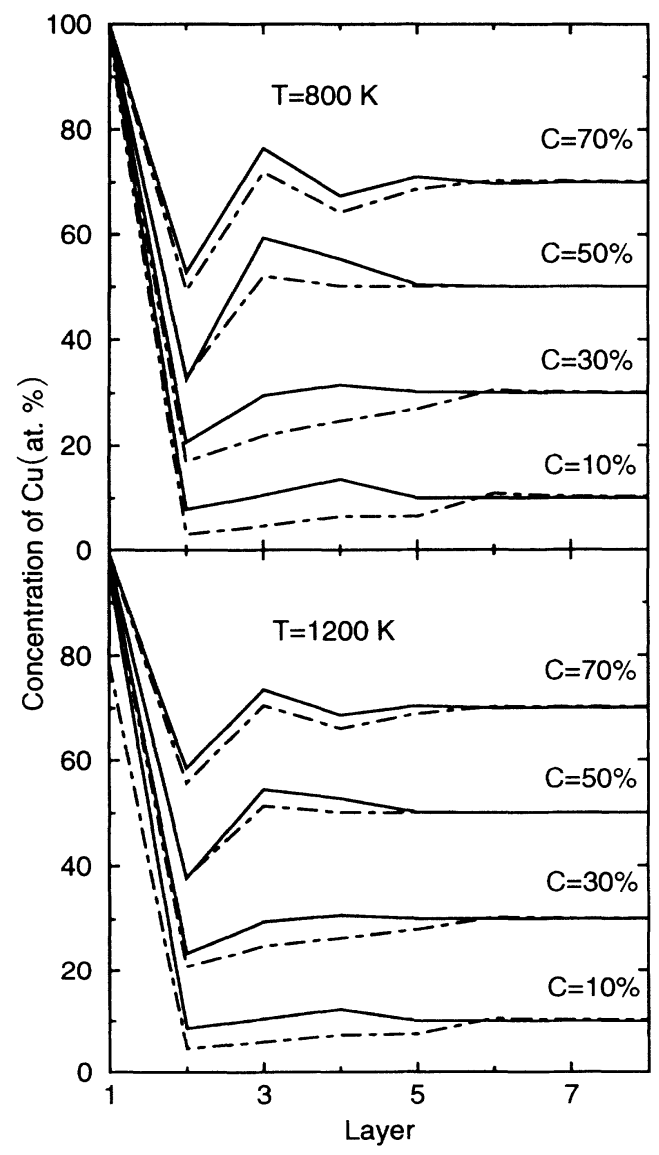

FIG. 6. Calculated equilibrium segregation profiles of the (001) surface of $\mathrm{Cu}-\mathrm{Ni}$ alloys for various bulk $\mathrm{Cu}$ concentrations at two temperatures. The full lines represent the results of the cluster variation method while the dot-dashed lines represent results obtained by the mean-field expression for the entropy. previous theoretical calculations in Fig. 7. In view of the challenge it is to determine segregation profiles from first principles we find that the calculations are in surprisingly good agreement. It is particularly gratifying to see that they all, in complete agreement with experiments, predict a strong segregation of $\mathrm{Cu}$ towards the surface and a rapid approach of the subsurface concentrations towards the bulk value.

The outstanding question is the existence of a $\mathrm{Cu}$ depletion in the first subsurface layer. In this respect the present results agree with the calculations based on the embedded atom method (EAM), ${ }^{11,15}$ but disagree with the GPM calculations. ${ }^{16,17}$ It appears that to settle this question, one cannot at present appeal to experiments since they are at variance with each other as discussed in the Introduction and summarized in Table I. One must therefore examine the approximations of the calculational techniques.

In the present technique the oscillatory behavior of the subsurface concentration is a direct consequence of the signs and the magnitudes of the calculated CWM one-site interactions which, however, in turn reflect the results of the direct LMTO-CPA surface-energy calculations. Hence the $\mathrm{Cu}$ depletion within the first subsurface layer cannot be attributed to some unwarranted approx-

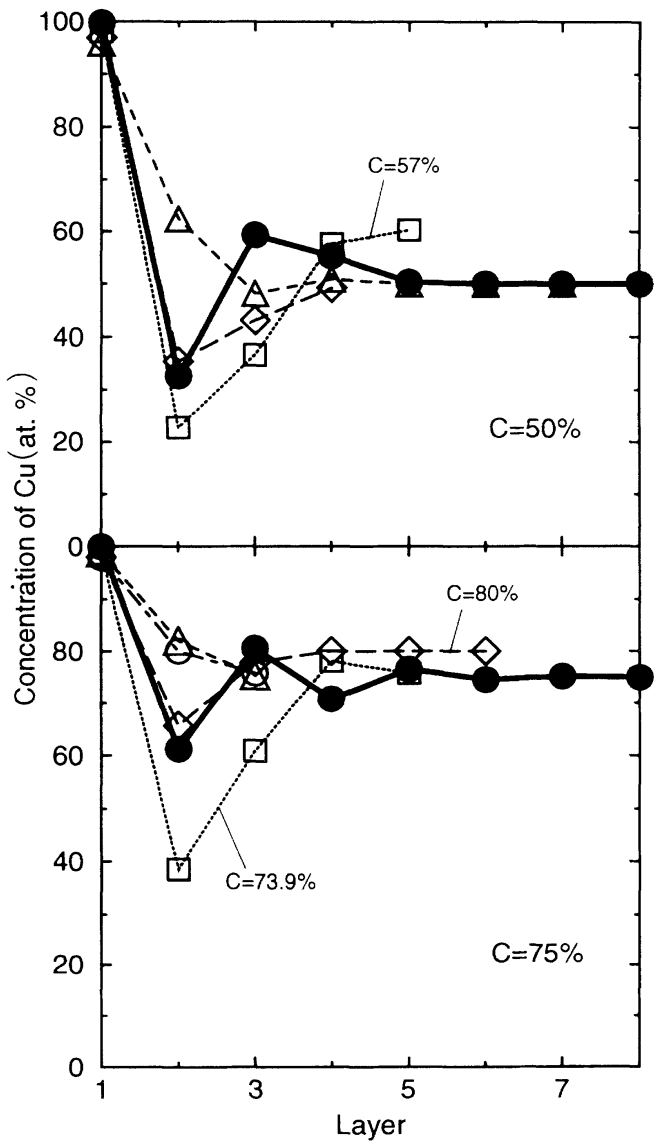

FIG. 7. Comparison of calculated segregation profiles. Filled circles, present results at $800 \mathrm{~K}$; open squares, EAM at $800 \mathrm{~K}$ (Ref. 11); open diamonds, EAM at $400 \mathrm{~K}$ (Ref. 15); open triangles, LMTO-CPA-GPM at $800 \mathrm{~K}$ (Ref. 17); open circles, TB-CPA-GPM at $700 \mathrm{~K}$ (Ref. 16). 
imation in the generalization of the Connolly-Williams method to the case of an alloy surface. In the present calculations, the main approximation, apart from the single-cite CPA, is the application of the atomic sphere approximation. However, the surface Green's-function tight-binding LMTO method on which the LMTO-CPA is based gives surface energies for metals in the noblemetal side of the Periodic Table which agree with those of full-potential calculations to within a few percent. ${ }^{32,55}$

It is perhaps significant that the calculations which predict an oscillatory segregation profile, i.e., LMTOCPA and EAM, are based on total energies rather than the one-electron energies used by GPM. However, to settle this issue clearly more work is needed.

\section{SUMMARY}

We have combined a number of calculational tools into a single scheme and used it to calculate physical properties of surfaces of random alloys at thermodynamic equilibrium from first principles. The scheme is based on the cluster variation method with interaction parameters determined by the Connolly-Williams method generalized to the case of a surface. The underlying total-energy calculations for a series of random alloys with specified bulk and surface-layer concentrations were carried out within the local density approximation to densityfunctional theory by means of the Green's-function surface LMTO-CPA method in the atomic sphere approximation.

The technique is applied in the calculation of the electronic structure and the surface segregation profile of the (001) surface of $\mathrm{Cu}-\mathrm{Ni}$ substitutional alloys. The layerprojected electronic state densities are presented for the alloys with different bulk and surface compositions. The band narrowing and the shift of the $d$-band center of gravity were analyzed within the tight-binding model. The calculated surface state densities are in good agreement with the experimental ultraviolet-photoelectron spectra with respect to the position, the bandwidth, and the relative intensities of the peaks.

The surface energy of a segregated $\mathrm{Cu}_{50} \mathrm{Ni}_{50}$ alloy is calculated by means of the LMTO-CPA method as a function of the surface and subsurface concentrations. These direct calculations show that the surface energy decreases with increasing $\mathrm{Cu}$ concentration in the surface layer and increasing $\mathrm{Ni}$ concentration in the subsurface layer. They strongly indicate that the segregation profile of the (001) surface of random $\mathrm{Cu}-\mathrm{Ni}$ alloys must be oscillatory.

The calculated Connolly-Williams interaction parameters for (001) surface indicate that the main contribution to the total energy from the surface and subsurface layer comes from the corresponding one-site interactions. In addition, the signs of the one-site interactions are such that one may expect strong segregation of $\mathrm{Cu}$ atoms into the surface layer and a weaker segregation of $\mathrm{Ni}$ atoms into the subsurface layer in complete agreement with the LMTO-CPA calculations.

The segregation profile of the (001) surface of random $\mathrm{CuNi}$ alloys is calculated by means of the cluster variation method and is found to be oscillatory for all bulk concentrations. This result disagrees with recent GPM calculations but is in complete agreement with total-energy EAM calculations.

\section{ACKNOWLEDGMENTS}

A.V.R., I.A.A., and H.L.S. would like to thank Börje Johansson and his group for creating a friendly environment and for hospitality in general. The Center for Atomic-Scale Materials Physics is sponsored by the Danish National Research Foundation. Part of the work was supported by grants from the Novo Nordisk Foundation and the Danish research councils through the Danish Center for Surface Reactivity.
${ }^{1}$ Interfacial Segregation, edited by W. C. Johnson and J. M. Blakely (American Society for Metals, Metals Park, OH, 1979).

${ }^{2}$ T. T. Tsong, Phys. Today 46 (5), 24 (1993).

${ }^{3}$ K. Watanabe, M. Hashiba, and T. Yamashina, Surf. Sci. 61, 483 (1976).

${ }^{4}$ F. J. Kuijers and V. Ponec, Surf. Sci. 68, 294 (1977).

${ }^{5}$ D. T. Ling, J. N. Miller, I. Landau, W. E. Spiecer, and D. M. Stefan, Surf. Sci. 74, 612 (1978).

${ }^{6}$ Y. S. Ng, T. T. Tsong, and S. B. McLane, Jr., Phys. Rev. Lett. 42, 588 (1979).

${ }^{7}$ Y. S. Ng, T. T. Tsong, and S. B. McLane, Jr., Surf. Sci. 84, 31 (1979).

${ }^{8}$ P. R. Webber, C. E. Rojas, and P. J. Dobson, Surf. Sci. 105, 20 (1981).

${ }^{9}$ N. Q. Lam, H. A. Hoff, H. Wiedersich, and L. E. Rehn, Surf. Sci. 149, 517 (1985).

${ }^{10}$ V. Kumar, Phys. Rev. B 23, 3756 (1981).
${ }^{11}$ S. M. Foiles, Phys. Rev. B 32, 7685 (1985).

${ }^{12}$ G. Treglia, B. Legrand, and P. Maugain, Surf. Sci. 225, 319 (1990).

13 J. Eymery and J. C. Jond, Surf. Sci. 231, 419 (1990).

${ }^{14}$ R. S. Jones, Phys. Rev. B 41, 3256 (1990).

${ }^{15}$ H. Y. Wang, R. Najafabadi, D. J. Srolovitz, and R. LeSar, Phys. Rev. B 45, 12028 (1992).

${ }^{16}$ H. Dreysse, L. T. Wille, and D. de Fontaine, Phys. Rev. B 47, 62 (1993).

${ }^{17}$ A. Pasturel, V. Drchal, J. Kudrnovsky, and P. Weinberger, Phys. Rev. B 48, 2704 (1993).

${ }^{18}$ P. J. Durham, R. G. Jordan, G. S. Sohal, and L. T. Wille, Phys. Rev. Lett. 53, 2038 (1984).

19 O. K. Andersen, Phys. Rev. B 12, 3060 (1975).

${ }^{20}$ O. Gunnarsson, O. Jepsen, and O. K. Andersen, Phys. Rev. B 27, 7144 (1983).

${ }^{21}$ H. L. Skriver, The LMTO Method (Springer-Verlag, Berlin, 1984). 
${ }^{22}$ O. K. Andersen and O. Jepsen, Phys. Rev. Lett. 53, 2571 (1984).

${ }^{23}$ O. K. Andersen, O. Jepsen, and D. Glötzel, in Highlights of Condensed-Matter Theory, edited by F. Bassani, F. Fumi, and M. P. Tosi (North-Holland, New York, 1985).

${ }^{24}$ O. K. Andersen, Z. Pawlowska, and O. Jepsen, Phys. Rev. B 34, 5253 (1986).

${ }^{25}$ W. R. L. Lambrecht and O. K. Andersen, Surf. Sci. 178, 256 (1986); and private communication.

${ }^{26}$ H. L. Skriver and N. M. Rosengaard, Phys. Rev. B 43, 9538 (1991).

${ }^{27}$ I. A. Abrikosov and H. L. Skriver, Phys. Rev. B 47, 16532 (1993).

28 J. W. D. Connolly and A. R. Williams, Phys. Rev. B 27, 5169 (1983).

${ }^{29}$ C. Wolverton, G. Ceder, D. de Fontaine, and H. Dreysse, Phys. Rev. B 48, 726 (1993).

${ }^{30}$ F. Ducastelle and F. Gautier, J. Phys. F 6, 2039 (1976).

${ }^{31}$ I. A. Abrikosov, A. V. Ruban, D. Ya. Kats, and Yu. H. Vekilov, J. Phys.: Condens. Matter 5, 1271 (1993).

${ }^{32}$ H. L. Skriver and N. M. Rosengaard, Phys. Rev. B 46, 7157 (1992).

${ }^{33}$ D. D. Johnson, D. M. Nicholson, F. J. Pinski, B. L. Gyorffy, and G. M. Stocks, Phys. Rev. B 41, 9701 (1990).

${ }^{34}$ V. Kumar and K. H. Bennemann, Phys. Rev. Lett. 53, 278 (1984).

${ }^{35}$ R. Kikuchi and J. W. Cahn, Acta Metall. 27, 1337 (1979).

${ }^{36}$ For a system without long-range order some terms in Eq. (10), e.g., $\sum_{i, k} L\left(v_{i k}^{\Lambda}\right)$ and $\sum_{i, l} L\left(v_{i l}^{\Lambda}\right)$ or $\sum_{i} L\left(x_{i}^{\Lambda}\right)$ and $\sum_{j} L\left(x_{j}^{\Lambda}\right)$, are equivalent. These terms have been explicitly kept in order to arrive at Eq. (12).

${ }^{37}$ J. M. Sanchez, F. Ducastelle, and D. Gratias, Physica 128A, 334 (1984).
${ }^{38}$ V. G. Vaks, N. E. Zein, and V. V. Kamyshenko, J. Phys. F 18, 1641 (1988).

${ }^{39}$ A. S. Steinberg, D. Ya. Kats, and B. A. Men, Phys. Status Solidi B 174, 9 (1992).

${ }^{40}$ V. G. Vaks, N. E. Zein, and V. V. Kamyshenko, J. Phys. Condens. Matter 1, 2115 (1989).

41 A. E. Carlsson, Phys. Rev. B 40, 912 (1989).

42 J. Kudrnovsky, I. Turek, V. Drchal, P. Weinberger, S. K. Bose, and A. Pasturel, Phys. Rev. B 47, 16525 (1993).

${ }^{43}$ K. Y. Yu, C. R. Helms, W. E. Spicer, and P. W. Chye, Phys. Rev. B 15, 1629 (1977).

${ }^{44}$ J. S. Faulkner, Prog. Mater. Sci. 27, 1 (1982).

${ }^{45}$ A. Gonis, Green Functions for Ordered and Disordered Systems (North-Holland, Amsterdam, 1992).

${ }^{46}$ A. Zangwill, Physics at Surfaces (Cambridge University Press, Cambridge, 1988).

47 J. Friedel, Ann. Phys. (N.Y.) 1, 257 (1976).

${ }^{48}$ J. R. Smith, J. G. Gay, and F. J. Arlinghaus, Phys. Rev. B 21, 2201 (1980).

49 O. Jepsen, J. Madsen, and O. K. Andersen, Phys. Rev. B 26, 2790 (1982).

${ }^{50}$ H. Krakauer, A. J. Freeman, and E. Wimmer, Phys. Rev. B 28, 610 (1983).

${ }^{51}$ E. Wimmer, A. J. Freeman, and H. Krakauer, Phys. Rev. B 30, 3113 (1984).

${ }^{52}$ I. A. Abrikosov, Yu. H. Vekilov, and A. V. Ruban, Phys. Lett. A 154, 407 (1991).

${ }^{53}$ J. Kudrnovsky, I. Turek, V. Drchal, P. Weinberger, N. E. Christensen, and S. K. Bose, Phys. Rev. B 46, 4222 (1992).

${ }^{54}$ Obtained from Fig. 2 of Ref. 17 and converted to Kelvin and the spin variable used in (16).

${ }^{55}$ M. Methfessel, D. Hennig, and M. Scheffler, Phys. Rev. B 46, 4816 (1992). 